\title{
MULTIPLICITY-FREE SPACES
}

\author{
VICTOR GUILLEMIN \& SHLOMO STERNBERG
}

\begin{abstract}
Introduction
Let $G$ be a Lie group. A unitary representation of $G$ on a Hilbert space, $H$, is called multiplicty-free if every irreducible representation of $G$ occurs in $H$ with multiplicity zero or one. It is easy to see that this is the case if and only if the ring of bounded $G$-invariant operators on $H$ is commutative. In this paper we will examine the symplectic analogue of this situation: Let $X$ be a symplectic manifold on which $G$ acts in a Hamiltonian fashion. If one thinks of the bounded operators on $H$ as "quantum observables" and the functions on $X$ as "classical observables" the analogue of the situation above is that the ring of $G$-invariant functions on $X$ be commutative with respect to Poisson-bracket. If this happens we will say that $X$ is multiplicity-free. We were led to the study of such manifolds by some questions in dynamical systems. Let $\Phi: X \rightarrow \mathfrak{g}$ * be the moment mapping. A function of the type $f \circ \Phi$, for $f: \mathfrak{g}^{*} \rightarrow \mathbf{R}$, is called collective (cf. [6]); a completely integrable system consisting of functions of this type is called a collective completely integrable system (see [12] or [20]). We noticed [12], that a necessary condition for $X$ to admit a collective completely integrable system is that it be multiplicity-free. We also proved that for certain groups, in particular for $U(n)$ and $O(n)$, this condition is sufficient as well.

This paper will consist of two parts. In part one we will study the local structure of multiplicity-free spaces in the neighborhood of a fixed coisotropic orbit. We will confine ourselves to the case where $G$ is compact and connected, though many of our results are true more generally. Our first main result will be that the problem of determining the local structure of such spaces, up to isomorphism, can be reduced to the special case where $X$ is a cotangent bundle. This reduction will involve two pieces of symplectic engineering which are of considerable interest in their own right. The first, the "induction" construction, is well known, but we will present it here in a somewhat unfamiliar guise. The second, the "cross-section" construction, was partly inspired by the material in
\end{abstract}

Received February 17, 1983, and, in revised form, July 6, 1983. 
$\S 9$ of Weinstein's paper [30] and by some unpublished remarks of his on "Gelfand models".

Suppose now that $M$ is a manifold on which $G$ acts and $X$ is its cotangent bundle. We will show that if the action of $G$ on $X$ is multiplicity-free, the action of $G$ on $M$ is transitive, i.e., $M=G / K, K$ being the stabilizer group of a base-point of $M$. We will next show that there is a simple necessary and sufficient condition for $X$ to be multiplicity-free in terms of the pair $G, K$. Let $\mathfrak{g}$ and $\mathbf{k}$ be the Lie algebras of $G$ and $K$ and let $\mathbf{k}^{0}$ be the annihilator of $\mathbf{k}$ in $\mathfrak{g}^{*}$. Then $X$ is multiplicity-free if and only if

$$
\begin{aligned}
& \text { for generic } \alpha \in \mathrm{g}^{*} \text { the } G \text {-orbit } G \cdot \alpha \\
& \text { intersects } k^{0} \text { in a finite union of } K \text {-orbits. }
\end{aligned}
$$

We will call a pair $G, K$ with this property a Gelfand pair. We will say a few words about the classification of Gelfand pairs below.

Part two of this paper will be concerned with the proof of the following

Theorem. Let $M$ be a manifold on which $G$ acts and let $H=L^{2}(M)=$ the space of $L^{2}$ half-densities on $M$. Then the representation of $G$ on $H$ is multiplicity-free if and only if the action of $G$ on $T^{*} M$ is multiplicity-free.

By Frobenius' theorem the representation of $G$ on $H$ is multiplicity-free if and only if every irreducible representation of $G$ contains at most one $K$-fixed vector. For $G$ an arbitrary simple compact connected Lie group the set of all closed subgroups $K$ with this property has been completely determined by Krämer in [18]. For instance if $G, K$ is a symmetric pair, $K$ has this property; however there are many examples not arising from symmetric pairs; for instance $S U(3)$ is a subgroup of $G_{2}$ with this property. In view of the theorem, Krämer's classification is also a classification of Gelfand pairs $G, K$ for which $G$ is simple. (Incidentally, the fact that symmetric pairs are Gelfand pairs in our sense was pointed out in [22].)

A crucial ingredient in Krämer's classification is the dimensional criterion

$$
\operatorname{dim} G \leqslant 2 \operatorname{dim} K+\operatorname{rank} G .
$$

Roughly speaking this criterion says that $K$ has to be a "large" subgroup of $G$, and one can show by inspection that simple groups do not have many large subgroups. Krämer's proof of $(* *)$ involves a rather complicated representation theoretic argument; however, we will show here that it is easily deduced from $(*)$.

The proof of the theorem involves a theorem-conjecture which is itself of interest. Let $X$ be a Hamiltonian $G$-space and let $A$ be the ring of collective functions on $X$. It is easy to see that the ring of $G$-invariant functions on $X$ is 
just the commutator, $A^{c}$, of $A$, with respect to Poisson bracket, in the ring of all functions on $X$. We conjecture that if $X$ is compact and connected, then $A^{c c}=A$, i.e. $A$ is its own double commutator. Otherwise stated, the ring $A$ of collective functions and the ring $A^{c}$ of invariant functions form a Howe pair in the Poisson algebra. We will prove a local form of this conjecture and suggest a method for proving it in general in $\$ 4$. An easy consequence of this conjecture is that if $X$ is multiplicity-free, then every $G$-invariant function on $X$ is of the form $f \circ \Phi$ where $f$ is a $G$-invariant function on $\mathrm{g}^{*}$. Assuming this we can give a rough idea of how the proof of the theorem goes: Let $Z(g)$ be the center of the universal enveloping algebra of $G$. There is a canonical morphism, $\rho$, of $Z(g)$ into the ring of $G$-invariant differential operators on $M$. We will show that $\rho$ extends to a morphism, $\rho^{\prime}$, of the ring, $Z^{\prime}(g)$, of bi-invariant pseudodifferential operators on $G$ into the ring of invariant pseudodifferential operators on $M$. Moreover, if $P \in Z^{\prime}(\mathrm{g})$ and $f$ is the restriction to $\mathrm{g}^{*}=T_{e}^{*}$ of its symbol, then the symbol of $\rho^{\prime}(P)$ is just $f \circ \Phi$. Now assume $T^{*} M$ is multiplicity-free. If the conjecture were true, then if $Q$ were a $G$-invariant pseudodifferential operator on $M$, the leading symbol of $Q$ would be of the form $f \circ \Phi$. Let $P_{0}$ be a bi-invariant pseudodifferential operator on $G$ whose leading symbol, restricted to $T_{e}^{*}$, is $f$. Then $\rho^{\prime}\left(P_{0}\right)$ has the same leading symbol as $Q$, so $Q-\rho^{\prime}\left(P_{0}\right)$ is of order less than $Q$. By repeated application of this argument it is possible to find $P \in Z^{\prime}(g)$ such that $Q-\rho^{\prime}(P)$ is of arbitrarily low order. Since $Z^{\prime}(g)$ is commutative, it follows that the ring of $G$-invariant pseudodifferential operators on $M$ is commutative modulo smoothing operators, and the ring of $G$-invariant differential operators is commutative on the nose. From this one can deduce, by a simple trick, that the representation of $G$ on $L^{2}(M)$ is multiplicity-free (see §6).

It turns out that this proof does not really require the full force of the double commutator theorem. It is enough to know that the double commutator theorem is true in the vicinity of a generic $G$-orbit, and this is easy to prove using the local structure theorems of $\S \S 1-3$ (see $\$ 4$ ).

Acknowledgements. The proof of the main theorem owes much to some conversations with Richard Melrose. Indeed our proof is a modified version of a proof he proposed, which was analytically more formidable but required less information about the local structure of multiplicity-free spaces. We would also like to thank Gerrit Heckman for calling our attention to Krämer's work, and Joe Wolf for suggesting the felicitous term: multiplicity-free, for the symplectic manifolds considered here. Finally we would like to thank the anonymous referees of this paper for the care with which they performed their task. 


\section{Symplectic induction}

The symplectic analogue of induced representation (and of the Frobenius reciprocity theorem) was introduced in [15] and studied in great detail in [8], see also [26] and [27]. In this section we shall describe a construction which, when applied to a special case gives this symplectic analogue of induced representations, but is a more general construction, including as well the symplectic analogue to certain kinds of holomorphic induction. We shall call our construction "symplectic induction". It is based on the theory of coisotropic embeddings as developed by Weinstein [25] and studied by Gotay [3]. We begin by recalling the salient features of this theory.

Let $X$ be a symplectic manifold with symplectic form $\omega$. A submanifold $\iota: Z \rightarrow X$ is called coisotropic if, at each point $z \in Z$ we have

$$
T Z_{z}^{\perp} \subset T Z_{z} .
$$

(Here the $\perp$ is with respect to the antisymmetric bilinear form $\omega_{z}$ on $T X_{z}$.) The spaces $T Z_{z}$ and hence $T Z_{z}^{\perp}$ all have the same dimension. Hence the spaces $T Z_{z}^{\perp}$ fit together to form a subbundle $T Z^{\perp}$ of $T Z$. This subbundle is well known to be integrable and hence defines a foliation on $Z$ which is just the null foliation of the restriction, $\sigma=\iota^{*} \omega$, to $Z$. The observation of Weinstein is that every manifold $Z$ with a closed two-form $\sigma$ of constant rank arises this way, and in a locally unique manner. More precisely,

Proposition 1.1 [25], [3], [28]. Let $Z$ be a manifold and $\sigma$ a closed two-form on $Z$ whose null spaces have constant dimension, and hence form a subbundle of $T Z$. Let $K$ be a compact group of diffeomorphisms of $Z$ which preserve $\sigma$. Then there exists a symplectic manifold, $X$, with symplectic form $\omega$, together with a symplectic $K$ action on $X$ and a $K$ equivariant embedding $\iota: Z \rightarrow X$ which makes $Z$ into a coisotropic submanifold of $X$ with $\iota^{*} \omega=\sigma$. If $X^{\prime}, \omega^{\prime}, \iota^{\prime}$ is a second such coisotropic embedding then there is a $K$ equivariant symplectic diffeomorphism, $\varphi: U \rightarrow U^{\prime}$ defined on neighborhoods $U$ and $U^{\prime}$ of $\iota(Z)$ and $\iota^{\prime}(Z)$ in $X$ and $X^{\prime}$ with $\iota^{\prime}=\varphi \circ \iota$.

Remark. This proposition is formulated and proved in the references cited without the $K$ action. But an examination of the proofs there shows that the $K$ equivariance can be built into their constructions and arguments.

We shall be interested in the following special case. Let $M$ be a symplectic manifold and let $P \stackrel{\pi}{\rightarrow} M$ be a principal $K$ bundle over $M$. If $\omega_{M}$ denotes the symplectic form on $M$ we shall take $Z=P$ and

$$
\sigma=\pi^{*} \omega_{M}
$$

The null leaves of $\sigma$ are just the fibers of $\pi$. Proposition 1.1 says that we can embed $P$ as a coisotropic submanifold of a symplectic $K$ space and that this 
embedding is locally unique (up to $K$ equivariant symplectic diffeomorphisms defined near $\iota(P))$. We shall now show that a choice of connection on $P$ will give us an explicit construction of $X$ which will show that the action of $K$ on $X$ is in fact Hamiltonian. The construction will also make $X$ into a fiber bundle over $M$. This fibration of $X$ over $M$ is not canonical however; it will depend on the choice of connection.

Let $\theta$ be a connection on $P$. Thus $\theta$ is a $k$-valued linear differential form on $P$. Set $Y=P \times k^{*}$. By abuse of language, we can think of $\theta$ as being a $k$-valued linear differential form on $Y$, depending only on the first factor. Let $\pi_{2}: P \times k^{*}$ denote projection onto the second factor, so that $\pi_{2}$ is a $k^{*}$-valued function on $Y$. Let $\langle$,$\rangle denote the pairing between k^{*}$ and $k$. Thus $\left\langle\pi_{2}, \theta\right\rangle$ is a scalar valued linear differential form on $Y$. Define the two-form $\omega$ on $Y$ by

$$
\omega=\pi^{*} \omega_{M}+d\left\langle\pi_{2}, \theta\right\rangle \text {. }
$$

It is clear that $\omega$ is closed. We let $K$ act on $Y=P \times k^{*}$ (diagonally) by the given action on $P$ and the coadjoint action on $k^{*}$. The transformation properties of a connection guarantee that $\left\langle\pi_{2}, \theta\right\rangle$, and hence $\omega$, is $K$ invariant. The injection

$$
\iota: P \rightarrow P \times k^{*}, \quad \iota(p)=(p, 0)
$$

is clearly $K$ equivariant and

$$
\iota^{*}(\omega)=\pi^{*} \omega_{M}=\sigma .
$$

We claim that $\omega$ is symplectic (i.e. nondegenerate) at all points of $\iota(P)$ and hence in some neighborhood of $\iota(P)$. Indeed, at a point $(p, 0)$ where $\pi_{2}=0$ we have

$$
d\left\langle\pi_{2}, \theta\right\rangle=\left\langle d \pi_{2} \wedge \theta\right\rangle .
$$

Also, the connection allows us to split $T P_{p}$ as $T P_{p}=H_{p}+V_{p}$, where $H_{P}$ and $V_{p}$ are the horizontal and vertical subspaces, and to identify $H_{p}$ with $T M_{\pi p}$ and $V_{p}$ with $k$. Thus we may identify $T Y_{(p, 0)}$ with $T M_{\pi p}+k+k^{*}$. Under this identification, the form $\omega_{(p, 0)}$ is given by $\left(\omega_{M}\right)_{p}$ on $T M_{\pi p}$ and the standard antisymmetric form on $k+k^{*}$, and the spaces $T M_{\pi p}$ and $k+k^{*}$ are orthogonal under $\omega_{(p, 0)}$. This proves that $\omega$ is symplectic at $\iota(P)$ and hence in some neighborhood of $\iota(P)$. In case $K$ and $M$ are compact we can be a little more precise about the neighborhood we choose. Indeed, for fixed $m_{0} \in M$, we can, since $K$ is compact, choose some neighborhood $\mathcal{V}_{0}$ of 0 in $k^{*}$ such that $\omega$ is symplectic at all points in $\pi^{-1}\left(m_{0}\right) \times \mathcal{V}_{0}$, and hence also at all points of $\pi^{-1}(m) \times \mathfrak{V}_{0}$, where $m$ is in some neighborhood of $m_{0}$. As $M$ is compact we may cover $M$ by finitely many such neighborhoods and let $\mathscr{V}$ be the intersection of the corresponding $\mathcal{T}_{0}$ 's. Thus, if $K$ and $M$ are compact we may find some neighborhood $\mathcal{V}$ of 0 in $k^{*}$ so that $\omega$ is symplectic on $P \times \mathcal{V}$. 
The action of $K$ on $P \times k^{*}$ is not only symplectic, it is Hamiltonian. Indeed, if $\xi \in k$ let $\xi_{P}$ be the corresponding vector field on $P$, and $\xi_{Y}$ the corresponding vector field on $Y$. Then

$$
i\left(\xi_{P}\right) \pi^{*} \omega_{M}=0 \text { and } i\left(\xi_{P}\right) \theta=\xi
$$

by the defining property of a connection. Hence by (1.1), the invariance of $\left\langle\theta, \pi_{2}\right\rangle$ and the preceding equation we have

$$
\begin{aligned}
i\left(\xi_{Y}\right) \omega & =i\left(\xi_{Y}\right) d\left\langle\theta, \pi_{2}\right\rangle \\
& =D_{\xi_{Y}}\left\langle\pi_{2}, \theta\right\rangle-d i\left(\xi_{Y}\right)\left\langle\pi_{2}, \theta\right\rangle=-d\left\langle\pi_{2}, \theta\right\rangle .
\end{aligned}
$$

Thus the action is Hamiltonian with moment map $\pi_{2}$. To summarize,

Proposition 1.2. Let $P \stackrel{\pi}{\rightarrow} M$ be a principal $K$ bundle over a symplectic manifold with form $\omega_{M}$. Let $\theta$ be a connection on $P$. Then (1.1) defines a symplectic form on some neighborhood of $\iota(P)$ in $P \times k^{*}$ and (1.3) holds. The action of $K$ on $P \times k^{*}$ is Hamiltonian with moment map $\pi_{2}$. If $K$ and $M$ are compact we can find some neighborhood $\mathcal{V}$ of 0 in $k^{*}$ so that $\omega$ is symplectic on $P \times \mathcal{V}$.

To relate Proposition 1.2 to the construction in [23], let $F$ be a Hamiltonian $K$ space with moment map $\Phi_{F}: F \rightarrow k^{*}$. Suppose that $\omega$ is symplectic on $P \times \mathcal{V}$, where $\mathcal{V}$ is a neighborhood of 0 in $k^{*}$ as in Proposition 1.2 and that $\Phi_{F}(F) \subset \mathcal{V}$. We can then form the space $X \times F$ with moment map $\Psi=\pi_{2}$ $\Phi_{F}$ and the Marsden-Weinstein reduced space $\Psi^{-1}(0) / K$. We can identify $\Psi^{-1}(0)$ with $P \times F$ since the $k^{*}$ component in $P \times k^{*} \times F$ is determined by $\pi_{2}=\Phi_{F}(f)$. It is then clear that (up to a sign) the form defined on $\Psi^{-1}(0)$ is the same as that given in [23], since $P \times F / K$ is the associated bundle, $F(P)$. In particular, the form on $F(P)$ is symplectic. Notice that this depended on $\Phi_{F}(F)$ being "small", i.e., in $\mathcal{V}$. The size of $\mathcal{V}$ depends, of course on $\theta$ and $\omega_{M}$. For compact $K$ and $M$ and fixed $\theta, \mathcal{V}$ can be made large by replacing $\omega_{M}$ by some large multiple, $a \omega_{M}$, where $a \gg 0$ is some large real number. This was observed by Weinstein (Remark (3) on p. 242 of [27]).

Now suppose that we are given a Lie subgroup $G \subset$ Aut $P$ and suppose that the induced action of $G$ on $M$ is Hamiltonian with moment map

$$
\Phi_{M}: M \rightarrow g^{*} \text {. }
$$

Also suppose that the connection $\theta$ is $G$-invariant. Then by letting $G$ act trivially on the $k^{*}$ component we get an action of $G$ on $P \times k^{*}$ which preserves $\omega$. (If $G$ and $K$ are compact then we could choose our symplectic neighborhood $X$ of $P \times\{0\}$ to be $G$-invariant.) The action of $G$ on $P \times k^{*}$ is in fact 
Hamiltonian. Indeed if $\zeta_{P}$ and $\zeta_{M}$ are the vector fields on $P$ and $M$ corresponding to $\zeta \in g$ then

$$
i\left(\zeta_{P}\right) d \pi^{*} \omega_{M}=\pi^{*} i\left(\zeta_{M}\right) \omega_{M}=-\pi^{*} d\left\langle\Phi_{M}, \zeta\right\rangle .
$$

Also, since $D_{\zeta_{P}} \theta=0$ and $\zeta_{P}$ has no $k^{*}$ component,

$$
i\left(\zeta_{P}\right) d\left\langle\pi_{2}, \theta\right\rangle=-d\left\langle\pi_{2}, i\left(\zeta_{P}\right) \theta\right\rangle .
$$

Now at each $p \in P$, the $k$-valued form $\theta$ defines a linear map of $g \rightarrow k$ given by

$$
\zeta \rightarrow\left(i\left(\zeta_{P}\right) \theta\right)_{p} .
$$

The transpose of this map is a linear map

$$
\Phi_{\theta, p}: k^{*} \rightarrow g^{*}
$$

and we can write the preceding equation as

$$
i\left(\zeta_{P}\right) d\left\langle\pi_{2}, \theta\right\rangle=-d\left\langle\Phi_{\theta}, \zeta\right\rangle,
$$

where $\Phi_{\theta}: P \times k^{*} \rightarrow g^{*}$ is given by

$$
\begin{aligned}
& \Phi_{\theta}(p, \beta)=\Phi_{\theta, p}(\alpha), \\
& \left\langle\zeta, \Phi_{\theta, p}(\beta)\right\rangle=\left\langle\theta,\left(i\left(\zeta_{P}\right) \theta\right)_{p}\right\rangle .
\end{aligned}
$$

Thus we have proved

Proposition 1.3. If $G \subset$ Aut $P$ preserves $\theta$ and induces a Hamiltonian action on $M$ with moment map $\Phi_{M}$, then the trivial action of $G$ on $k^{*}$ gives a Hamiltonian action of $G$ on $P \times k^{*}$ with moment map

$$
\Phi_{G}=\pi^{*} \Phi_{M}+\Phi_{\theta},
$$

where $\Phi_{\theta}$ is given by (1.4).

Let us now give two rather different applications of Proposition 1.3. In the first of these we shall take $M=\vartheta$ to be an orbit of $G$ in $g^{*}$ and $K=G_{\alpha}$ to be the isotropy subgroup of some point $\alpha$ of $\theta$. We shall take $P=G$ regarded as a principal $k$-bundle over $\theta=G / K$, with $G$ acting as automorphisms via left multiplication. A $G$-invariant connection on $P$ is given by the choice of an Ad $K$ invariant complement, $p$, to $k$ in $g$. (This determines the horizontal space of the connection at the identity $e \in G=P$ and $G$ invariance then determines it everywhere. The Ad $K$ invariance is required for it to be a connection.) If $K$ is compact we can always make this choice by putting an $\operatorname{Ad} K$ invariant metric on $g$. (For the case that $G$ is compact we shall study this situation in more detail in $\S 2$ and show that then there is a canonical choice of $p$.) 
The splitting $g=k+p$ gives a splitting $g^{*}=k^{*}+p^{*}$ and hence a linear map $l=l_{p}$ of $k^{*} \rightarrow g^{*}$. Then (1.5) becomes

$$
\Phi_{G}(a, \beta)=a \cdot(\alpha+l(\beta)) .
$$

Indeed, this is exactly the content of (1.5) at the point $a=e$, and hence by the $G$ equivariance of the moment map it is true everywhere.

As a second illustration of the construction of Proposition 1.3, let us consider the following situation: let $\rho: Q \rightarrow N$ be a principal $K$ bundle where $N$ is any differentiable manifold, not necessary symplectic. The group $K$ has a natural Hamiltonian action on the cotangent bundle, $T^{*} Q$. Inside $T^{*} Q$ there is a $K$ invariant coisotropic submanifold, $P$, consisting of those covectors which vanish when evaluated on any vertical tangent vector of $Q$. A covector in $T^{*} Q$ belongs to $P$ if and only if it is of the form $d \rho_{q}^{*}(\kappa)$ where $\kappa \in T^{*} N_{n}$ with $n=\rho(q)$. We thus have a natural projection $\pi: P \rightarrow T^{*} N$, where $\pi\left(d \rho_{q}^{*}(\kappa)\right)=$ $\kappa$. It is easy to check that this makes $P$ into a principal $K$ bundle over $T^{*} N$ and the diagram

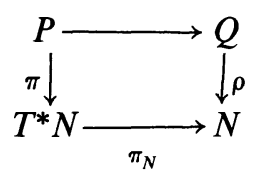

commutes, where $\pi_{N}: T^{*} N \rightarrow N$ is the standard projection of the cotangent bundle onto the base and the map from $P \rightarrow Q$ is the restriction of the standard projection $T^{*} Q \rightarrow Q$ to $P$. Thus we can regard $P$ as being the pullback of the bundle $Q \rightarrow N$ to $T^{*} N$, in other words, $P=\pi_{N}^{\#}(Q)$. We could start with this abstract definition of $P$ as a pulled back bundle (cf. [23]) over $M=T^{*} N$ and use the embedding $\iota: P \rightarrow T^{*} Q=X$ to give the coisotropic embedding. This construction was used by Weinstein, [26], who observed that a connection on $Q$ giving a splitting of $T^{*} Q$ into horizontal and vertical components makes $T^{*} Q$ into a fiber bundle over $T^{*} N$. (This fibration is the same as that given more generally in Proposition 1.2 if we take the connection there to be the pullback of the connection on $Q$ to $P$.) If $N=G / K$ is a homogeneous $G$ space, then $G$ acts in Hamiltonian fashion on $T^{*} N$, and the construction of Proposition 1.3 applied to any Hamiltonian $K$ space gives rise to a Hamiltonian $G$ space. This is the symplectic analogue of the induced representation, cf. [15] and [8].

\section{The cross-section construction}

Let $G$ be a connected Lie group, $X$ a symplectic manifold on which $G$ acts in a Hamiltonian fashion, and $\Phi: X \rightarrow \mathbb{S S}^{*}$ the moment map associated with this action. Let $O$ be a coadjoint orbit and $\alpha \in O$. The cross-section theorem says 
that if $Y$ is a submanifold of $g^{*}$ containing $\alpha$ and

$$
\mathrm{g}^{*}=T_{\alpha} O \oplus T_{\alpha} Y
$$

then there exists a neighborhood, $\mathscr{U}$, of $\alpha$ in $Y$ such that $\Phi^{-1}(\mathcal{Q})$ is a symplectic submanifold of $X$. A proof of this theorem can be found, for instance, in $[12, \S 1]$. If $G$ happens to be a compact Lie group this theorem can be sharpened by making a particularly nice choice of $Y$. To see this we need first to recall some elementary facts about compact connected Lie groups. Fix a point $\alpha \in \mathrm{g}^{*}$ and let $K$ be the stabilizer group of $\alpha$ in $G$. It is clear that $K$ is closed and one can also show rather easily that $K$ is connected. Let $M$ be the center of $K$, and consider in $\mathrm{g}^{*}$ the subspaces

$$
k^{\#}=\text { the elements of } g^{*} \text { stabilized by } M
$$

and

$$
m^{\#}=\text { the elements of } g^{*} \text { stabilized by } K \text {. }
$$

Clearly $m^{\#} \subseteq k^{\#}$ and $\alpha \in m^{\#}$. It is also clear that the coadjoint action of $K$ on $\mathrm{g}^{*}$ preserves $k^{\#}$. We will prove the following standard facts about this situation.

Theorem 2.1. (1) The orbit, $O$, through $\alpha$ intersects $k^{\#}$ transversally at $\alpha$ and at this intersection (2.1) is satisfied, with $Y=k^{\#}$.

(2) For points $\beta \in k^{\#}$ near $\alpha$ the stabilizer of $\beta$ in $G$ is contained in $K$.

(3) The canonical projection $\mathrm{g}^{*} \rightarrow k^{*}$ maps $k^{\#}$ bijectively onto $k^{*}$.

(4) The canonical projection $\mathrm{g}^{*} \rightarrow m^{\#}$ maps $m^{\#}$ bijectively onto $m^{*}$.

(5) Every coadjoint orbit in $\mathrm{g}^{*}$ intersects $k^{\#}$ in a finite number of $K$ orbits.

Before proving these assertions we will make a few remarks: Parts (1) and (2) of the theorem simply say that $k^{\#}$ is a slice for the action of $G$ on $\mathrm{g}^{*}$ in the sense of Koszul, Palais, etc. Part (3) says that $k^{*}$ can be canonically imbedded in $\mathrm{g}^{*}$ and as such is a complementary space to

$$
k^{0}=\left\{f \in \mathrm{g}^{*},\langle f, \xi\rangle=0 \text { for all } \xi \in k\right\} .
$$

Part (4) is a similar assertion about $m^{*}$.

Proof. Fix a positive definite $G$-invariant bilinear form on $\mathfrak{g}^{*}$. Associated with this form one gets a bijective $G$-equivariant map $\rho: \mathfrak{g}^{*} \rightarrow \mathfrak{g}$. We claim

$$
\rho\left(k^{\#}\right)=k \text { and } \rho\left(m^{\#}\right)=m .
$$

To see this let $\xi=\rho(\alpha)$. By definition

$$
k=\{\eta \in \mathfrak{g},[\xi, \eta]=0\} .
$$

Since $[\xi, \xi]=0, \xi$ is in $k$ and, therefore, by (2.3) $\xi$ is in the center, $m$, of $k$. Since $\rho\left(m^{\#}\right)$ is the subset of $g$ stabilized by $K,\left[\xi, \rho\left(m^{\#}\right)\right]=0$, so $\rho\left(m^{\#}\right)$ is contained in $k$ by (2.3) and hence is equal to the center of $k$, i.e. $\rho\left(m^{\#}\right)=m$. 
Similarly, since $\rho\left(k^{\#}\right)$ is the subset of $g$ stabilized by $M,\left[\xi, \rho\left(k^{\#}\right)\right]=0$, so $\rho\left(k^{\#}\right)$ is contained in $k$. On the other hand, it is obvious that $\rho\left(k^{\#}\right) \supset k$; so $\rho\left(k^{\#}\right)=k$. This proves $(2.2)$.

Parts (3) and (4) of the theorem follow immediately from (2.2). To prove part (1) let $p=\left(k^{\#}\right)^{0}$ in $\mathrm{g}$. Then

$$
\mathrm{g}=p \oplus k \quad \text { (orthonormal decomposition). }
$$

Moreover, $\operatorname{ad}(\xi): \mathfrak{g} \rightarrow \mathfrak{g}$ preserves (2.4) mapping $k$ into zero and $p$ bijectively onto itself. This shows that $p=[\xi, \mathrm{g}]=$ the tangent space to the orbit through $\xi$, proving (1). To prove (2), let $\eta$ be an element of $k$. Then ad( $\eta$ ) preserves (2.4). Moreover, since $\operatorname{ad}(\xi): p \rightarrow p$ is bijective, $\operatorname{ad}(\eta): p \rightarrow p$ is bijective if $\eta$ is sufficiently close to $\xi$. Thus the centralizer of $\eta$ in $\mathrm{g}$ is contained in $k$. Finally to prove (5) let $t$ be a Cartan subalgebra of $g$ containing $\xi$. Then by (2.3) $t$ is contained in $k$; so it is also a Cartan subalgebra of $k$. Let $\eta_{1}$ and $\eta_{2}$ be elements of $k$ on the same $G$-orbit. Then they have $K$-conjugates $\eta_{1}^{\prime}$ and $\eta_{2}^{\prime}$ in $t$. However, if two elements of $t$ are conjugate by $G$ they are Weyl-group conjugate (see for instance, Humphreys [14]). Thus every $G$-orbit intersects $k$ in at most $n K$-orbits where $n$ is the cardinality of the Weyl group. q.e.d.

As above fix a positive definite inner product on $\mathrm{g}^{*}$ which is $G$-invariant and let

$$
B_{\varepsilon}(\alpha)=\left\{v \in k^{\#},\|v-\alpha\|<\varepsilon\right\} .
$$

It is clear that $B_{\varepsilon}(\alpha)$ is $K$-invariant. Moreover, by part (5) of the theorem, for $\varepsilon$ sufficiently small every $G$-orbit in $\mathrm{g}^{*}$ intersecting $B_{\varepsilon}(\alpha)$ intersects $B_{\varepsilon}(\alpha)$ in exactly one $K$-orbit. Now let $X$ be a symplectic manifold on which $G$ acts in a Hamiltonian fashion and let $\Phi: X \rightarrow \mathrm{g}^{*}$ be the associated moment map. Let

$$
W=\Phi^{-1}\left(B_{\varepsilon}(\alpha)\right) .
$$

For $\varepsilon$ sufficiently small, $W$ is a symplectic submanifold of $X$. We note the following properties of $W$.

Proposition 2.2. (a) $W$ is $K$-invariant.

(b) The action of $K$ on $W$ is Hamiltonian and the associated moment map is just the map $\Phi: W \rightarrow k^{\#}$ composed with the identification $k^{\#} \rightarrow k^{*}$.

(c) Every G-orbit in $X$ which intersects $W$ intersects $W$ in a single $K$-orbit.

Proof. Parts (a) and (b) are obvious. To prove (c) let $p_{1}$ and $p_{2}$ be $G$-related points in $W$. Then $\Phi\left(p_{1}\right)$ and $\Phi\left(p_{2}\right)$ are $G$-related in $B_{\varepsilon}(\alpha)$, so they are $K$-related. Hence without loss of generality we can assume $p_{1}=g p_{2}$ and $\Phi\left(p_{1}\right)=\Phi\left(p_{2}\right) \in B_{\varepsilon}(\alpha)$. Thus $g$ is in the stabilizer of $\Phi\left(p_{1}\right)=\Phi\left(p_{2}\right)$, so by part (2) of the theorem $g \in K$. q.e.d. 
Let $X_{0}$ be the union of all points $p$ such that $G p$ intersects $W . X_{0}$ is an open $G$-invariant subset of $X$. Moreover the ring of $G$-invariant functions on $X_{0}$ is, by restriction, isomorphic with the ring of $K$-invariant functions on $W$ and this isomorphism is a morphism of Poisson algebras so we have proved that $X_{0}$ is multiplicity-free if and only if $W$ is multiplicity-free.

We will show shortly that the manifold, $X_{0}$, can be reconstructed from $W$ in a canonical way. Before we do this, however, let us show how the cross-section construction can be used to give an alternative version of an induction construction presented in $\S 1$. (The material below is essentially due to Alan Weinstein, [30, §9].)

Let $\Phi_{R}: T^{*} G \rightarrow \mathrm{g}^{*}$ be the moment map associated with the right action of $G$ on $T^{*} G$ and consider $Z=\Phi_{R}^{-1}\left(k^{\#}\right)$. If we identify $T^{*} G$ with $G \times \mathrm{g}^{*}$ by means of the right action of $G$ then $\Phi_{R}$ is just the projection of $G \times \mathrm{g}^{*}$ onto $\mathrm{g}^{*}$; so $Z=G \times k^{\#}$. By the cross-section theorem, $Z$ is a symplectic submanifold of $T^{*} G$ at least in the $G \times K$ invariant neighborhood

$$
Z_{\alpha}=G \times B_{\varepsilon}(\alpha)
$$

of $G \times\{\alpha\}=\Phi_{R}^{-1}(\alpha)$. Now in $\S 1$ we considered the orbit, $O$, through $\alpha$ and the principal $K$-bundle

$$
\pi: G \rightarrow O, \quad g \rightarrow g \alpha .
$$

The splitting $\mathrm{g}=p \oplus k$ with $p=\left(k^{\#}\right)^{0}$ defines a connection on this bundle and we showed in $\$ 1$ how to use this connection to define a symplectic structure on a neighborhood

$$
Z_{0}=G \times B_{\varepsilon}(0)
$$

of $G \times\{0\}$ in $G \times k^{*}$. It is easy to see that the symplectic structure on $Z_{\alpha}$ given by the cross-section construction and the symplectic structure on $Z_{0}$ given by the induction construction are essentially the same. In fact let $\tau: Z_{0} \rightarrow Z_{\alpha}$ be the map $\tau(g, \beta)=(g, \alpha+\beta)$. This map is $G \times K$ equivariant since $\alpha$ is $K$-fixed; and it is not hard to show that it is a $G \times K$ equivariant symplectomorphism.

Coming back to $X_{0}$ and $W$ we will now indicate how $X_{0}$ can be reconstructed from $W$ in a canonical way. The moment mapping associated with the Hamiltonian action of $K$ on $Z_{\alpha}$ is just the projection map

$$
Z_{\alpha}=G \times B_{\varepsilon}(\alpha) \rightarrow B_{\varepsilon}(\alpha)
$$

by Proposition 2.2, so we can form the product of Hamiltonian $K$-spaces, $Z_{\alpha} \times W^{-}$, and reduce with respect to the zero orbit in $k^{*}$. This is exactly the symplectic induction construction discussed in $§ 1$. Let us denote the resulting space by $X_{1}$. 
Theorem 2.3. $\quad X_{0}$ and $X_{1}$ are isomorphic as Hamiltonian G-spaces.

Proof. As an abstract set

$$
X_{1}=(G \times W) / K \text {. }
$$

Map $W$ into $X_{1}$ by the mapping

$$
i: W \rightarrow X_{1}, \quad w \rightarrow \text { equivalence class of }(e, w) .
$$

It is easy to check that $i$ is an imbedding and is $K$-equivariant. There is a unique way of extending $i$ to a $G$-equivariant map of $X_{0}$ onto $X_{1}$. Namely if $x \in X_{0}$ pick an element $g \in G$ and $w \in W$ such that $x=g w$ and set $\sigma(x)=$ $g i(w)$. Let us show that this unambiguously defines a map $\sigma: X_{0} \rightarrow X_{1}$. Suppose we also have $x=g_{1} w_{1}$. Then $g^{-1} g_{1} w_{1}$ and $w_{1}$ are on the same $G$-orbit in $W$, so they are on the same $K$-orbit, i.e. there exists $k_{0} \in K$ such that $k_{0} g^{-1} g$ stabilizes $w_{1}$. This implies that $k_{0} g^{-1} g$ stabilizes the point $\Phi\left(w_{1}\right)$ in $B_{\varepsilon}(\alpha)$, and, therefore, has to be in $K$. Thus there exists an element $k \in K$ such that

$$
g_{1}=g k \text {. }
$$

In particular $g_{1} w_{1}=g k w_{1}=g w$, or $k w_{1}=w$. Since $i$ is $k$-equivariant, $i(w)=$ $k i\left(w_{1}\right)$, so

$$
g i(w)=g k i\left(w_{1}\right)=g_{1} i\left(w_{1}\right)
$$

by (2.5). This proves that $\sigma$ is well defined. Since $\sigma$ is smooth on $W$ and $G$-equivariant, it is smooth everywhere. It is easy to check that it is a symplectomorphism at all points $w \in W$ and so, by $G$-equivariance, at all points of $X_{0}$.

\section{The cotangent bundle case}

Let $X$ be a Hamiltonian $G$-space and $\Phi: X \rightarrow g^{*}$ the associated moment mapping. In $\$ 2$ of [12] we proved that the three following conditions are equivalent.

I. $X$ is multiplicity-free.

II. For generic points $p \in X$ the orbit of $G$ through $p$ is coisotropic.

III. For generic points, $\alpha$, in the image of $\Phi, \Phi^{-1}\left(\theta_{\alpha}\right)$ is a finite union of $G$-orbits, $\theta_{\alpha}$ being the coadjoint orbit through $\alpha$.

Using these conditions we will show first of all how the problem of classifying multiplicity-free spaces can be reduced to the special case of $X$ a cotangent bundle (locally in the vicinity of a coisotropic orbit), and secondly we will analyze in detail the cotangent case. 
Suppose that for $p \in X$ the orbit $G$ through $p$ is coisotropic. Let $\alpha=\Phi(p)$ and let $K$ be the stabilizer group of $\alpha$ in $G$. Let $B_{\varepsilon}(\alpha)$ be the ball of radius $\varepsilon$ in $k^{\#}$ centered at $\alpha$ and let $W=\Phi^{-1}\left(B_{\varepsilon}(\alpha)\right)$. The $G$-orbit through $p$ intersects $W$ in the $K$-orbit, $M$, through $p$ and, since $W$ is symplectic and this intersection is transversal, $M$ is a coisotropic submanifold of $W$. On the other hand $M$ is an isotropic submanifold of $W$. To see this let $\Phi_{W}$ be the moment map associated with the action of $K$ on $W$. We showed in $\S 2$ that $\Phi_{W}=\Phi 1 W$; so in particular $\Phi_{W}(p)=\alpha$. Since $\alpha$ is $K$-fixed, it follows that $\Phi_{W}$ is constant on $M$. On the other hand the Hamiltonian vector fields associated with the components of $\Phi_{W}$ span the tangent space to $M$ at each point of $M$; so the symplectic form of $W$ restricted to $M$ vanishes. Since $M$ is both isotropic and coisotropic, we have proved

Theorem 3.1. The $K$-orbit, $M$, through $p$ in $W$ is a Lagrangian submanifold of $W$.

By the Weinstein-Darboux theorem there exists a $K$-invariant neighborhood, $W_{0}$, of $M$ in $W$, a $K$-invariant neighborhood, $\mathscr{\vartheta}_{0}$, of the zero section in $T^{*} M$ and a $K$-equivariant symplectomorphism $\Psi: W_{0} \rightarrow \mathcal{Q}_{0}$ such that $\Psi 1 M$ is the standard imbedding of $M$ as the zero section in $T^{*} M$. By Theorem 2.3 we can locally reconstruct $X$ in the vicinity of the $G$-orbit through $p$ by applying the symplectic induction procedure to $W_{0}$. Thus we have reduced the problem of classifying multiplicity-free spaces to the cotangent case. Before taking up this case we mention an application of Theorem 3.1. Suppose $\alpha$ is a regular element of $\mathrm{g}^{*}$. Then $K=T=$ a Cartan subgroup of $G$, and $T$ is the stabilizer of every point $\beta \in B_{\varepsilon}(\alpha)$; so by Theorem 3.1 the orbits of $T$ in $W$ are Lagrangian submanifolds of $W$. In other words the action of $T$ on $W$ is completely integrable. We already mentioned in the introduction one tie-in between the theory of completely integrable systems and multiplicity-free spaces. The cross-section construction furnishes another tie-in. (For an interesting example of this construction, see [12].)

Now let $M$ be a manifold on which $G$ acts and let $X=T^{*} M$. We will first show

Lemma. If the action of $G$ on $X$ is multiplicity-free, the action of $G$ on $M$ is transitive.

Proof. If the action were not transitive, we could find a $G$-invariant function $f$ on $M$ with $d f$ not identically zero. Equip $M$ with a $G$-invariant Riemannian metric and let $\Xi$ be the gradient vector field associated with $f$. The flow on $M$ generated by $\Xi$ lifts to a Hamiltonian flow on $X$. Let $H$ be the Hamiltonian function generating this flow, let $\pi: X \rightarrow M$ be the cotangent fibration and let $F=\pi^{*} f$. Then

$$
\{F, H\}=\pi^{*} D_{\Xi} f=\pi^{*}\|d f\|^{2} .
$$


By assumption the right-hand side is nonzero; so the ring of $G$-invariant functions on $X$ is noncommutative. q.e.d.

Now fix a basepoint $m_{0} \in M$ and let $K$ be the stabilizer group of $m_{0}$. Then $M \cong G / K$ and $T_{m_{0}} \cong \mathrm{g} / k$.

Let $k^{0}$ be the annihilator of $k$ in $\mathrm{g}^{*}$. The moment mapping associated with the action of $K$ on $X$

$$
\Phi: X \rightarrow \mathfrak{g}^{*}
$$

is completely determined by the fact that it is $G$-equivariant and is equal to the canonical mapping

$$
T_{m_{0}}^{*} \cong(g / k)^{*}=k^{0}
$$

on $T_{m_{0}}^{*}$. Let $\theta$ be a coadjoint orbit in $\mathrm{g}^{*}$. If $X$ is multiplicity-free, $G$ acts transitively on the connected components of $\Phi^{-1}(\theta)$ by (3.1)III. Since $G$ acts transitively on $M$ this means that $K$ acts transitively on the connected components of $\Phi^{-1}(\theta) \cap T_{m_{0}}^{*}$ or, in view of (3.2),

$$
\vartheta \cap k^{0} \text { is a finite union of } K \text {-orbits. }
$$

Thus we have proved that $X$ is multiplicity-free if and only if the orbit $\theta_{\alpha}$ through a generic point $\alpha \in k^{0}$ satisfies (3.3). An equivalent way of formulating this condition is as follows. Let

$$
f_{1}, \cdots, f_{r}, \quad r=\operatorname{rank} G,
$$

be the Casimir functions on $g^{*}$. By Chevalley's theorem these functions generate the ring of $G$-invariant functions on $\mathfrak{g}^{*}$ (see [13]). Since $G$ is a compact group, the $G$-invariant functions separate orbits, so

(3.5) $\alpha$ and $\beta$ are the same $G$-orbits $\Leftrightarrow f_{i}(\alpha)=f_{i}(\beta), \quad i=1, \cdots, r$,

Let $\mathcal{Q}$ be the subset of $k^{0}$ consisting of all points $\alpha \in k^{0}$ such that the $G$-orbit through $\alpha$ intersects $k_{0}$ in a finite union of $K$-orbits. It is obvious that $U$ is a $K$-invariant subset of $k^{0}$ and by (3.3) $X$ is multiplicity-free if and only if $\mathscr{Q}$ is a "generic" set, i.e., an open, dense subset of $k^{0}$. Let $g_{i}=f_{i} 1$ थ. Then by (3.5) the level sets

$$
g_{i}=c_{i}, \quad i=1, \cdots, r,
$$

are finite unions of $K$-orbits no matter what the values of the $c_{i}$ 's are. In particular choose a point $\alpha \in \mathcal{U}$ such that the space spanned by $\left(d g_{i}\right)_{\alpha}$, $i=1, \cdots, r$, has maximal dimension, say $s \leqslant r$. Without loss of generality one can assume that $d g_{1}, \cdots, d g_{s}$ are linearly independent at $\alpha$. Then the $K$-orbit through $\alpha$ is locally defined by the equations

$$
g_{i}=g_{i}(\alpha), \quad i=1, \cdots, s ;
$$


so it is of codimension $s$ in $k^{0}$. Since its dimension is less than or equal to the dimension of $K$ we get

$$
\operatorname{dim} K \geqslant \operatorname{dim} k^{0}-s \geqslant \operatorname{dim} g-\operatorname{dim} k-r,
$$

i.e.,

$$
\operatorname{dim} G \leqslant 2 \operatorname{dim} K+\operatorname{rank} G .
$$

This is the inequality of Krämer mentioned in the introduction.

\section{The double commutator theorem}

Let $X$ be a symplectic manifold on which $G$ acts in a Hamiltonian fashion and let $\Phi: X \rightarrow \mathrm{g}^{*}$ be the associated moment map. Let $\mathbb{Q}$ be the ring of collective functions on $X$; i.e., functions of the form $f \circ \Phi, f$ being a smooth function on $\mathfrak{g}^{*}$. Note that $\mathcal{Q}$ is closed under Poisson brackets since $\{f \circ \Phi, g \circ \Phi\}=\{f, g\} \circ \Phi$. Let $\mathscr{B}$ be the ring of $G$-invariant functions on $X$. It is easy to see that $\mathscr{B}=\mathbb{Q}^{c}=$ the commutator of $\mathscr{Q}$ in the Poisson algebra. Indeed, a function, $H$, on $X$ commutes with all functions of the form above if and only if it commutes with the $\xi$ th component of the moment map for all $\xi \in \mathrm{g}$. Thus, since the Hamiltonian vector field associated with the $\xi$ th component of the moment map is the vector field $\xi^{\#}$ induced by $\xi$ on $X$, this implies that for all $\xi \in \mathfrak{g}, D_{\xi^{*}} H=0$, i.e., $H$ is $G$-invariant.

We will now prove a local converse of this result. Let $थ$ be an open subset of $X$ such that, on $\mathcal{Q}, \Phi$ has constant rank and the level surfaces

$$
\{x \in \mathcal{Q}, \Phi(x)=c\}
$$

are connected. Let $F$ be a smooth function on $थ$. We will show that if $\{F, H\}=0$ for all $H \in \mathscr{B}$ then there exists a smooth function, $f$, on $\mathrm{g}^{*}$ such that $F=f \circ \Phi$.

Proof. Let $p \in \mathcal{Q}$ and let $c=\Phi(p)$. The tangent space to the level surface $\{x \in \mathcal{Q}, \Phi(x)=c\}$ at $p$ is the symplectic orthocomplement of the tangent space to the $G$-orbit through $p$ (see $[12, \S 1]$ ). Therefore, if $H$ is a $G$-invariant function and $\xi_{H}$ its Hamiltonian vector field,

$$
\left\langle d H_{p}, v\right\rangle=\omega\left(\xi_{H}, v\right)=0
$$

for all $v$ tangent to the orbit through $p$. This shows that $\xi_{H}$ is tangent to the level surface at $p$, and it is clear that all tangent vectors to the level surface are of this form. Hence if $\{H, F\}=0$ for all such $H, F$ is locally constant along the level surface. If the level surface is connected, $F$ takes on a single value, 
$f(c)$, on the level surface; and, therefore, there is a function, $f$, on the image of Q such that $F=f \circ \Phi$. It is clear that one can extend $f$ to the ambient space. q.e.d.

We conjecture that if $G$ is compact and connected, then, globally,

$$
\mathbb{Q}=\mathscr{B}^{c}=\mathbb{Q}^{c c}=\text { its own double commutator. }
$$

We will present two sets of evidence for this conjecture. First of all let $V$ be an open subset of $g^{*}$ such that all points $\alpha \in V$ are regular values of $\Phi$. It is clear from the preceding arguments that $\mathscr{Q}=\mathbb{Q}^{c c}$ on $\Phi^{-1}(V)$ if and only if the level surfaces

$$
X_{\alpha}=\{x \in X, \Phi(x)=\alpha\}
$$

are connected for all $\alpha \in V$. It was proved by Atiyah [2] that this is true if the group, $G$, is abelian; and from recent work of Kirwan [16] and Ness [2] on Morse-theoretic properties of the "Yang-Mills" function, $\langle\Phi, \Phi\rangle$, one gets the impression that it is very likely to be true in the nonabelian case as well.

The other evidence for this conjecture comes from singularity theory, Suppose for simplicity that $G$ is abelian. Let $p$ be a singular point of $\Phi$, and let us consider the worst case scenario when $d \Phi_{p}=0$. Then $p$ is a fixed point of $G$. It was proved by us [9] and independently by Arms, Marsden \& Moncrief [1] that in this case the moment map, $\Phi$, and the moment map associated with the linear symplectic action of $G$ on the tangent space at $p$ are locally conjugate by a symplectomorphism. In particular there exists a Darboux coordinate system $x_{1}, y_{1}, \cdots, x_{n}, y_{n}$ centered at $p$ such that the coordinates of the moment map are of the form

$$
\phi_{\alpha}=\sum_{i=1}^{n} \kappa_{i}^{(\alpha)}\left(x_{i}^{2}+y_{i}^{2}\right), \quad \alpha=1, \cdots, N=\operatorname{dim} G,
$$

the $\kappa_{i}^{(\alpha)}$ 's being constants. In fact the $\kappa_{i}^{(\alpha)}$ 's are just the components of the weights of the representation of $G$ on $T_{p}$; so by choosing an appropriate basis of $\mathfrak{g}$, one can arrange that the $\kappa_{i}^{(\alpha)}$ 's are integers. As further evidence for the double commutator conjecture we will prove

Proposition 4.1. The double commutator of the set $\left\{\phi_{1}, \cdots, \phi_{N}\right\}$ is identical with the set of collective functions, $f\left(\phi_{1}, \cdots, \phi_{N}\right)$ with $f \in C^{\infty}\left(\mathbf{R}^{N}\right)$.

Proof. Since $x_{1}^{2}+y_{1}^{2}, \cdots, x_{n}^{2}+y_{n}^{2}$ commute with the functions (4.1), a function in the double commutator has to commute with these functions and therefore has to be of the form $f\left(x_{1}^{2}+y_{1}^{2}, \cdots, x_{n}^{2}+y_{n}^{2}\right)$, where $f=f\left(t_{1}, \cdots, t_{n}\right)$ is a $C^{\infty}$ function on $\mathbf{R}^{n}$. To prove the theorem it is enough to show that

$$
\sum m_{i} \frac{\partial f}{\partial t_{i}}=0
$$


whenever

$$
\sum m_{i} \kappa_{i}^{(\alpha)}=0, \quad \alpha=1, \cdots, N .
$$

Moreover, since the $\kappa_{i}^{(\alpha)}$ 's are integers it is enough to verify (4.2) for $n$-tuples of integers, $m_{1}, \cdots, m_{n}$, satisfying (4.3). Suppose $f$ is of the form $f\left(x_{1}^{2}+y_{1}^{2}, \cdots, x_{n}^{2}\right.$ $\left.+y_{n}^{2}\right)$. Then for $g=g\left(x_{1}, y_{1}, \cdots, x_{n}, y_{n}\right)$,

$$
\frac{1}{2}\{f, g\}=\sum\left(x_{i} \frac{\partial g}{\partial y_{i}}-y_{i} \frac{\partial g}{\partial x_{i}}\right) \frac{\partial f}{\partial t_{i}} \text {. }
$$

Introducing polar coordinates, $x_{i}+\sqrt{-1} y_{i}=r_{i} e^{\sqrt{-1} \theta_{i}}$, we get

$$
\frac{1}{2}\{f, g\}=\sum \frac{\partial g}{\partial \theta_{i}} \frac{\partial f}{\partial t_{i}} \text {. }
$$

Now let $m_{1}, \cdots, m_{n}$ be a sequence of integers and let

$$
g=r_{1}^{\left|m_{1}\right|} e^{\sqrt{-1} m_{1} \theta_{1}} \cdots r_{n}^{\left|m_{n}\right|} e^{\sqrt{-1} m_{n} \theta_{n}}
$$

Then (4.4) reduces to

$$
\sqrt{-1} g \sum m_{i} \frac{\partial f}{\partial t_{i}}
$$

so if $m_{1}, \cdots, m_{n}$ satisfy (4.3), $g$ is in the commutator of $\left\{\phi_{1}, \cdots, \phi_{N}\right\}$, and hence if $f$ is in the double commutator it satisfies (4.2). q.e.d.

Suppose now that $X$ is multiplicity-free. This means that $Q^{c}$ is commutative, and hence $\mathbb{Q}^{c} \subseteq \mathbb{Q}^{c c}$. Therefore, if the double commutator theorem were true every $G$-invariant function, $H$, on $X$ could be written in the form

$$
H=f \circ \Phi
$$

for some smooth function, $f$, on $\mathrm{g}^{*}$. If we replace $f$ by its translate by any element of $G$ this does not change the left-hand side of (4.5); so by averaging with respect to Haar measure we can assume that $f$ is $G$-invariant. In other words the double commutator conjecture would imply that the ring of $G$ invariant functions on $X$ is identical with the ring of collective functions of the form (4.5) with $f$ also G-invariant. We will conclude this section by proving a local form of this result: It was proved in $[12, \S 1]$ that if $X$ is a Hamiltonian $G$-space, then for generic points $p \in X$ the stabilizer group of $p$ is a normal subgroup of the stabilizer group of $\Phi(p)$ and the quotient group is abelian. If $X$ is multiplicity-free, then, as we pointed out in $\S 3$, for generic points $p \in X$ the orbit of $G$ through $p$ is coisotropic. Let $p$ be a point of $X$ which is generic in both these senses. We will prove

Proposition 4.2. There exists a G-invariant neighborhood, $V$, of $p$ in $X$ such that every G-invariant function on $X$ is of the form (4.5) on $V, f$ being a G-invariant function on $\mathrm{g}^{*}$. 
Proof. We will first show that by using the cross-section construction of $\S 2$, the theorem can be reduced to a much simpler theorem: Let $\alpha=\Phi(p)$ and let $K$ be the stabilizer group of $\alpha$ in $G$. Let $B_{\varepsilon}(\alpha)$ be the ball of radius $\varepsilon$ about $\alpha$ in $k^{\#}$, with $\varepsilon$ small, and let $W=\Phi^{-1}\left(B_{\varepsilon}(\alpha)\right)$. Finally let $X_{0}$ be the set of points in $X$ whose $G$-orbits intersect $W$. We pointed out in $\S 2$ that there is a one-to-one correspondence between $G$-invariant functions on $X_{0}$ and $K$-invariant functions on $W$. Since the moment map, $\Phi_{W}$, is just the restriction to $W$ of the moment map, $\Phi$, collective functions on $X_{0}$ restrict to collective functions on $W$. Conversely, it is clear that if $F$ is a $K$-invariant collective function on $W$, its unique $G$-invariant extension to $X_{0}$ is also collective. Thus to prove the double commutator conjecture for $X_{0}$ it is enough to prove it for $W$. Let $M$ be the $K$-orbit through $p$. We pointed out in $\S 3$ that in the vicinity of $M, X$ and $T^{*} M$ are isomorphic as Hamiltonian $K$-spaces, so we get a further simplification of our problem: It is enough to prove the double commutator conjecture for $T^{*} M$. By assumption the stabilizer group of $p, G_{p}$, is normal in $K$ and $K / G_{p}$ is abelian; therefore, setting $H=K / G_{p}$ we are ultimately reduced to proving the double commutator conjecture for the cotangent bundle of $H$. This is easy. We leave for the reader to check that in this case all three rings $\mathcal{Q}, \mathbb{Q}^{c}$ and $\mathbb{Q}^{c c}$ can be identified with the ring of smooth functions on $h^{*}$.

\section{When is an induced representation multiplicity-free?}

Let $M$ be a compact manifold on which $G$ acts and let $L^{2}(M)$ be the Hilbert space of $L^{2}$ half-densities. For each $g \in G$ denote by $T_{g}$ the operator

$$
T_{g} f(m)=f\left(g^{-1} m\right) \text {. }
$$

Clearly (5.1) defines a unitary representation of $G$ on $L^{2}(M)$. If $h$ is a $C^{\infty}$ function on $G$ then by integrating $h(g) T_{g}$ with respect to Haar measure we get an operator

$$
T_{h}=\int h(g) T_{g} d \mu
$$

on $L^{2}(M)$, and the map which associates $T_{H}$ to $h$ is a representation of the convolution algebra of $G$ on $L^{2}(M)$. In certain instances one can define (5.2) for distributional functions, $h$. For instance if $h$ is a distribution supported at the identity element, then the operator, convolution by $h$, is a left invariant differential operator on $G$. In this case the map $h \rightarrow T_{h}$ is the usual representation of the universal enveloping algebra of $G$ as differential operators on $M$. Modulo some "cleanness" assumptions about the action of $G$ on $M$ one can also define $T_{h}$ for distributions whose singular support is the identity element 
of $G$. For instance let us assume that $G$ acts transitively on $M$. Let $\varrho_{e}$ be the set of Lagrangian distributions on $G$ with singular support at $\{e\}$. Given $h \in \mathcal{L}_{e}$ the operator, convolution with $h$, is a left invariant pseudodifferential operator on $G$, and, conversely every left invariant pseudodifferential operator is of this form; so $\mathcal{L}_{e}$ is a subalgebra of the convolution algebra of distributional functions on $G$. We will show shortly that if $h \in \mathcal{L}_{e}$, then $T_{h}$ is well defined and is a pseudodifferential operator on $M$. In other words if we denote the ring of pseudodifferential operators on $M$ by $\Psi O P_{M}$ there is a morphism of rings

$$
T: \mathcal{L}_{e} \rightarrow \Psi O P_{M} \text {. }
$$

We will also show that if the symbol of $h$ on $T_{e}^{*}=\mathrm{g}^{*}$ is the function, $f$, the symbol of $T_{h}$ is $f \circ \Phi, \Phi$ being the moment map restricted to $T^{*} M-0$. In other words the symbols, $\sigma\left(T_{h}\right)$ and $\sigma(h)$ are related by

$$
\sigma\left(T_{h}\right)=\sigma(h) \circ \Phi \text {. }
$$

To prove these statements we need to recall a few facts about symplectic group actions: Given a symplectic manifold, $X$, and a Hamiltonian action of $G$ on $X$, let $\Phi$ be the associated moment map, and identify $T^{*} G$ with $G \times \mathrm{g}^{*}$ by means of the right action of $G$ on itself. Consider the subset

$$
\Gamma_{\Phi}=\{(g, \eta, x, g x), x \in X, g \in G, \eta=\Phi(x)\},
$$

of the product space $T^{*} G \times X \times X$. This set is a canonical relation, the so-called moment relation (see [7] or [28].) If $X=T^{*} M-0,(5.5)$ is just the canonical relation associated with the operator

$$
h \in C^{\infty}(G) \rightarrow T_{h} .
$$

To be more specific, by Schwartz's kernel theorem, $T_{h}$ can be viewed as a distributional function on $M \times M$; so (5.6) can be viewed as an operator from $C^{\infty}(G)$ to $C^{-\infty}(M \times M)$. As such it is a Fourier integral operator and its associated canonical relation is (5.5). (A proof of these remarks can be found in [11].) Now if $\Lambda=T_{e}^{*}, \Gamma$ and $\Lambda$ intersect transversally if (and only if) $G$ acts transitively on $M$, and, in this case, $\Gamma \circ \Lambda$ is the identity canonical relation in $\left(T^{*} M-0\right) \times\left(T^{*} M-0\right)$. It follows from standard facts about the composition of Lagrangian distributions that if $h$ is a Lagrangian distribution on $G$ with singular support equal to the identity element, then $T_{h}$ is an operator whose Schwartz kernel is a Lagrangian distribution on $M \times M$ with singular suppport on the diagonal; in other words, $T_{h}$ is a pseudodifferential operator. This establishes (5.3), and (5.4) is a simple consequence of standard facts about the composition of symbols of F.I.O's.

Next we observe that the discussion above can be "microlocalized" (for the microterminology below see; for instance, [4]): Let $X=T^{*} M-0$, let $\mathscr{Q}$ be a 
$G$-invariant open conic subset of $g^{*}-0$ and let $V$ be a $G$-invariant open conic subset of $\Phi^{-1}(\mathscr{U})$. We will denote by $\Re_{थ}$ the ring of Lagrangian microfunctions with support on $\mathscr{U}$ in $T^{*} G-0$ (the ring structure being given by convolution), and we will denote by $\mathfrak{R} O P_{V}$ the ring of microdifferential operators on $V$. Then for $h \in \mathfrak{N}_{\mathscr{Q}}, T_{h}$ is well defined as an element of $\mathfrak{N} O P_{V}$; and so we get a morphism of rings, microlocalizing (5.3),

$$
T: \Re_{\mathscr{Q}} \rightarrow \mathfrak{K} O P_{V} \text {. }
$$

Moreover, at the symbolic level the relation between the symbol of $h$ and the symbol of $T_{h}$ is still given by (5.4). Notice also that (5.7) maps the subring $\left(\Re_{\mathcal{Q}}\right)^{G}$ of Ad $G$-invariant elements of $\Re_{ひ}$ into the subring $\left(\mathfrak{N}_{O} O P_{V}\right)^{G}$ of $G$-invariant elements of $\Re O P_{V}$. In other words there is a morphism of rings

$$
T:\left(\Re_{\mathcal{Q}}\right)^{G} \rightarrow\left(\mathscr{N}_{O} O P_{V}\right)^{G} \text {. }
$$

Suppose now that $X$ is multiplicity-free. Let $p$ be a generic point of $X$ in the sense of Proposition 4.2 with $\Phi(p) \in$ Q . We will prove

Proposition 5.1. For all sufficiently small $G$-invariant neighborhoods, $V$, of $p$ (5.8) is surjective.

Proof. Let $P$ be in $\left(\mathfrak{T} O P_{V}\right)^{G}$ and let $F$ be its symbol. since $F$ is $G$ invariant there exists, by Proposition 4.2, a $G$-invariant function, $f$, on $\mathcal{U}$ such that $F=f \circ \Phi$. Let $h_{0}$ be a Lagrangian microfunction on $\mathcal{U}$ whose leading symbol is $f$. By averaging over $G$ one can arrange that $h_{0} \in\left(\Re_{Q}\right)^{G}$. Since $T_{h_{0}}$ and $P$ have the same leading symbol, $T_{h_{0}}-P$ is of order one less than $P$. By repeating this argument with $P$ replaced by $T_{h_{0}}-P$, etc., one eventually obtains an $h \in\left(\mathscr{T}_{\mathscr{Q}}\right)^{G}$ with $T_{h}=P$. q.e.d.

Now let $h_{1}$ and $h_{2}$ be elements of $\left(\Re_{\mathcal{Q}}\right)^{G}$ and let $\tilde{h}_{1}$ and $\tilde{h}_{2}$ be distributions such that the microfunctions associated with them are $h_{1}$ and $h_{2}$. By averaging $\tilde{h}_{1}$ and $\tilde{h}_{2}$ with respect to Haar measure we can arrange that they are $G$-invariant. Then convolution by $\tilde{h}_{1}$ and convolution by $\tilde{h}_{2}$ are bi-invariant operators on $C^{\infty}(G)$. By the Peter-Weyl theorem the representation of $G \times G$ on $L^{2}(G)$ is multiplicity-free; so these operators have to commute. Thus $\tilde{h}_{1}$ and $\tilde{h}_{2}$ commute with respect to convolution. This proves that the ring $\left(\Re_{Q}\right)^{G}$ is commutative, and, hence by Proposition 5.1 that the ring $\left(\mathscr{N} O P_{V}\right)^{G}$ is commutative. Since the set of generic points $p \in X$ is dense in $X$, the ring $\left(\Re\left(O P_{X}\right)^{G}\right.$ is commutative. This proves

Theorem 5.2. If $X$ is multiplicity-free, the ring of G-invariant pseudodifferential operators on $M$ is commutative modulo smoothing operators.

As a corollary; we get

Theorem 5.3. If $X$ is multiplicity-free, the ring of G-invariant differential operators on $M$ is commutative. 
We will use Theorem 5.3 to prove, in the next section, our main theorem, namely

Theorem 5.4. The representation of $G$ on $L^{2}(M)$ is multiplicity-free iff the action of $G$ on $X$ is multiplicity-free.

\section{An elementary lemma}

We will prove in this section the following elementary result, for which we unfortunately could not find a reference in the literature.

Lemma. Let $M$ be a compact connected real analytic manifold. Let $f_{1}, \cdots, f_{k}$ be a real analytic functions and $h_{1}, \cdots, h_{k} C^{\infty}$ functions on $M$. If the $f_{i}$ 's are linearly independent there exists a differential operator, $Q$, with $C^{\infty}$ coefficients such that $Q f_{i}=h_{i}, i=1, \cdots, k$.

Before we prove this theorem a few remarks are in order.

1. It is clear that the theorem is not true with the $f_{i}$ 's $C^{\infty}$. For instance if $f_{1}$ were to vanish on an open set, $h_{1}$ would have to vanish on the same open set.

2. It is enough to prove the theorem locally. Indeed, suppose that for each point $p \in M$ we can find an open neighborhood, $\mathcal{U}$, of $p$ and a differential operator, $Q_{\text {थ }}$, on $\mathcal{Q}$ such that $Q_{\mathcal{Q}} f_{i}=h_{i}$ on $\mathcal{Q}$ for $i=1, \cdots, k$. Cover $M$ by such neighborhoods and select a partition of unity subordinate to this covering, say $\left\{\rho_{\text {थ }}\right\}$. Then if $Q=\sum \rho_{\text {थ }} Q_{\text {थ }}, Q f_{i}-h_{i}, i=1, \cdots, k$, on all of $M$.

3. It is obviously sufficient to prove the theorem with $h_{1}=\cdots=h_{k-1}=0$ and $h_{k}=1$.

4. Let $Q$ be the ring of germs of real-analytic functions defined in a neighborhood of the origin in $\mathbf{R}^{n}$ and let $\mathscr{Q}$ be the ring of differential operators with coefficients in $\mathcal{Q}$. Given a finite set $\mathscr{F}=\left\{f_{1}, \cdots, f_{k}\right\} \subseteq \mathbb{Q}$, let $\mathscr{F}^{c} \subseteq \mathcal{D}$ be the left ideal consisting of $P \in \mathscr{Q}$ for which

$$
P f_{i}=0, \quad i=1, \cdots, k .
$$

To prove statement 3 it is enough to prove

Sublemma. Suppose $f \in \mathscr{Q}$ and $P f=0$ for all $P \in \mathscr{F}^{c}$. Then $f$ is a linear combination of $f_{1}, \cdots, f_{k}$.

Let us see how to reduce statement 3 to this sublemma. Given linearly independent functions $f_{1}, \cdots, f_{k} \in \mathbb{Q}$ we want to find $Q \in \mathcal{Q}$ such that $Q f_{1}=$ $\cdots=Q f_{k-1}=0$ and $Q f_{k}=1$. Let $\mathcal{F}=\left\{f_{1}, \cdots, f_{k-1}\right\}$. By the sublemma there exists $P \in \mathscr{F}^{c}$ such that $P f_{k}$ is not identically zero. This means that there exists a combination of derivatives such that $D^{\alpha} P f_{k}(0) \neq 0$. Set $g=\left(D^{\alpha} P f\right)^{-1}$ in $Q$ and let $Q=g D^{\alpha} P$. 
We will prove the sublemma by induction on $k$. If $k=1$ then, with $g=f_{1}$, $\mathscr{F}^{c}$ contains the operators

$$
Q_{g}^{i}=g \frac{\partial}{\partial x_{i}}-\frac{\partial g}{\partial x_{i}}, \quad i=1, \cdots, n,
$$

and it is clear that if $h \in \mathbb{Q}$ is annihilated by these operators it is a constant multiple of $g$.

Suppose now that the theorem is true for $k-1$ and let us prove it for $k$. Let $\mathcal{F}^{\prime}=\left\{f_{1}, \cdots, f_{k-1}\right\}$ and let $f=f_{k}$. For each $P \in\left(\mathcal{F}^{\prime}\right)^{c}$ let $f_{P}=P f$, and let $Q_{P}^{i}$ be the operator (6.1) with $g=f_{P}$. Consider the collection of operators

$$
Q_{P}^{i} P, \quad P \in\left(\mathscr{F}^{\prime}\right)^{c}, i=1, \cdots, n,
$$

and the collection of operators

$$
f_{P} Q-f_{Q} P, \quad P, Q \in\left(\mathscr{F}^{\prime}\right)^{c} .
$$

Note that all these operators belong to $(\mathscr{F})^{c}$. Suppose now that $h \in \mathbb{Q}$ has the property that

$$
P h=0 \text { for all } P \in \mathscr{F}^{c} .
$$

Conditions (6.2) and (6.4) imply that for all $P \in\left(\mathscr{F}^{\prime}\right)^{c}, P h$ is a constant multiple, $a_{P}$, of $f_{P}$. The conditions (6.3) imply that

$$
a_{Q} f_{P} f_{Q}=a_{P} f_{P} f_{Q}
$$

and finally condition (6.4) implies that $P h=0$ if $f_{P}=0$. Thus there exists a fixed constant $a$ such that for all $P \in\left(\mathscr{F}^{\prime}\right)^{c}, P(h-a f)=0$. By induction this implies that $h-a f$ is a linear combination of $f_{1}, \cdots, f_{k-1}$. This proves the sublemma and so also the lemma.

We will now prove Theorem 5.4: The second order Casimir element in the universal enveloping algebra of $G$ defines a selfadjoint second order elliptic operator, $\Delta$, on $M$.

If $B: L^{2}(M) \rightarrow L^{2}(M)$ is $G$-invariant, it commutes with $\Delta$, so it maps each of the eigenspaces of $\Delta$ into itself. Let $V$ be an eigenspace of $\Delta$. Then by the lemma there exists a differential operator, $Q$, such that $B=Q$ on $V$. By averaging with respect to Haar measure one can make $Q G$-invariant; so we have proved that every bounded operator, $B$, on $L^{2}(M)$ is equal to a $G$-invariant differential operator on $V$. Therefore, by Theorem 5.3, the ring of bounded $G$-invariant operators is a commutative ring of operators on $V$. Since the $V$ 's span $L^{2}(M)$ it follows that the ring of bounded $G$-invariant operators on $L^{2}(M)$ is itself commutative.

We have proved that if $X$ is multiplicity-free then the representation of $G$ on $L^{2}(M)$ is multiplicity-free. The converse result is much easier: If $L^{2}(M)$ is 
multiplicity-free, the $G$-invariant pseudodifferential operators on $M$ commute so their symbols Poisson-commute, and from this one easily deduces that the ring of $G$-invariant functions on $X$ is commutative.

\section{Concluding remarks}

1. Let $G$ be a compact connected Lie group. Let $\rho_{c}$ be a Hamiltonian action of $G$ on a symplectic manifold and $\rho_{q}$ a unitary representation of $G$ on a Hilbert space. Suppose that, in an appropriate sense, $\rho_{c}$ and $\rho_{q}$ are the classical and quantum descriptions of the same underlying physical system. The result of $\$ 5$ suggests the following conjecture: $\rho_{q}$ is multiplicity-free if and only if $\rho_{c}$ is multiplicity-free. To test this conjecture we examined an example which is slightly trickier than the example of $\$ 5$. Namely let $K$ be a closed subgroup of $G$ and let $\gamma$ be a $K$-fixed element of $k^{*}$. If $\gamma$ is integral it defines a character of $K$. Let $M=G / K$ and let $L$ be the line bundle over $M$ associated with this character. Let $X$ be the $L$-shifted cotangent bundle of $M$ in the sense of Weinstein [25] and Kostant [17]. There is a simple criterion for $X$ to be multiplicity-free similar to (3.3); namely, for generic elements $\alpha \in k^{0}$

The $G$-orbit through $\alpha+\gamma$ intersects $k^{0}+\gamma$ in a finite number of $K$-orbits.

Denote by $\tilde{\gamma}$ the induced representation of $G$ associated with $\gamma$. By modifying the proof of Theorem 5.4 one can prove that $\tilde{\gamma}$ is multiplicity-free if and only if the Hamiltonian action of $G$ on $X$ is multiplicity-free. In fact the only modification one needs to make in the previous proof is to use, in place of the usual symbol calculus on $T^{*} M-0$, the $L$-shifted symbol calculus on $X$ in the sense of Kostant.

2. It would also be interesting to test the conjecture above for $X$ a Kähler manifold. The quantized action would then be on the "Fock space" of $X$. For instance the conjecture is true for the standard representation of $U(n)$ on Fock space. It is also "generically" true for compact Kähler manifolds, [10], and for compact strictly pseudoconvex domains [11].

3. The results of $\$ 3$ suggest a procedure for constructing multiplicity-free representations. Let $G \supset K \supset L$ be a triple of compact groups such that:

(i) $G / K$ is a generalized flag manifold and

(ii) $K, L$ is a Gelfand pair.

Let $\mathcal{K}_{0}=L^{2}(K / L)$ and let $\mathcal{H}$ be the Hilbert space obtained by holomorphically inducing $\mathcal{H}_{0}$ to $G$. It would be interesting to know whether there is a quantum analogue of the structure theorem in $\S 3$ : i.e., is every multiplicity-free 
representation of $G$ "locally" of the form above, whatever that means. Unfortunately the representation of $G$ on $\mathcal{H}$ itself is usually not multiplicity-free. There is an action of the Weyl group which identifies equivalent irreducible subrepresentations.

4. It would be interesting to know whether there is also a quantum analogue of the double commutator conjecture. For instance let $M$ be a compact manifold on which $G$ acts. For simplicity assume that the action of $G$ is transitive. Let $(\Psi O P G)^{G}$ be the ring of left-invariant pseudodifferential operators on $G$ and $\Psi O P M$ the ring of pseudodifferential operators on $M$. As we pointed out in $\S 5$ there is a canonical morphism of rings

$$
\Psi:(\Psi O P G)^{G} \rightarrow \Psi O P M
$$

which can be regarded as the quantum analogue of the moment map. Let $\mathfrak{Q}_{q}$ be its image. It is easy to see that the commutator, $\mathbb{Q}_{q}^{c}$, of $\mathbb{Q}_{q}$ in the ring of all pseudodifferential operators on $M$ is just the subring of $G$-invariant pseudodifferential operators. Is $\mathbb{Q}=\mathbb{Q}^{c c}$ ? Clearly this conjecture is stronger than the classical double commutator conjecture for $X=T^{*} M$. In fact the classical conjecture is equivalent to the assertion $\mathscr{Q}=\mathbb{Q}^{c c}$, modulo smoothing operators. For some ramifications of the double-commutator conjecture see [15, §3].

5. It would be interesting to see whether the Krämer classification of Gelfand pairs could be simplified by using in place of the dimensional criterion (3.6) the more precise information given by (3.3).

6. As we pointed out in $\S 1$, a manifold with a closed two-form of constant rank can be imbedded coisotropically in a symplectic manifold, and locally this imbedding is unique up to symplectomorphism. Does there exist such a canonical imbedding similar to the canonical isotropic imbedding described by Weinstein in [29]? Even if not is there a canonical way of obtaining symplectic reduced spaces for Hamiltonian $K$-spaces without the restrictions imposed in $\$ 1$ vi2 that the image of the moment map lie in a small neighborhood of the origin? For example, Formula 1 , makes sense for arbitrary $K$-orbits and produces a symplectic induced space (i.e., a $G$-orbit) even though the general construction requires that the $K$-orbit be small compared with the $G$-orbit.

7. At the quantum level we only understand the analogue of the construction in $\S 1$ in two special cases: ordinary induction and holomorphic induction. We would like to know if there is a quantum analogue in general.

8. Using the results of $\S \S 1$ and 2 one can prove a "local canonical form" theorem for the moment map $\Phi: X \rightarrow g^{*}$ at any point $p$ in $X$. Indeed, one can show that $\Phi$ is determined, up to isomorphism in a $G$-invariant neighborhood of $p$ by the following three pieces of data:

(i) the value $\alpha=\Phi(p)$, 
(ii) the stabilizer group, $G_{p}$, and

(iii) the linear isotropy representation of $G_{p}$ on the tangent space at $p$.

Proof. By the cross-section theorem one can reduce to the case where $\alpha$ is $G$-fixed. Then the $G$-orbit, $M$, through $p$ is isotropic. A $G$-equivariant version of the isotropic imbedding theorem of Weinstein [29] says that the action of $G$ on a sufficiently small $G$-invariant neighborhood of $M$ is determined, up to isomorphism, by the action of $G$ on the symplectic normal bundle $E \rightarrow M$. However $E$, in this case, is the homogeneous bundle associated with the isotropy action of $G_{p}$ on the symplectic normal space to $M$ at $p$. q.e.d.

From this result one can derive all the known local canonical form theorems for the moment map, e.g. [1], [2], [5]-[12]. We will elaborate on this point in a future article.

\section{References}

[1] J. Arms, J. Marsden \& V. Moncrief, Symmetries and bifurcations of the momentum mapping, Comm. Math. Phys. 78 (1981) 455-478.

[2] M. Atiyah, Convexity and commuting Hamiltonians, Bull. London Math. Soc. 14 (1982) 1-15.

[3] M. Gotay \& J. Nester, Presymplectic manifolds and the Dirac-Bergmann theory of constraints, J. Math. Phys. 19 (1978) 2388-2399.

[4] V. Guillemin, M. Kashiwara \& T. Kawai, Seminar on micro-local analysis, Annals of Math. Studies No. 93, Princeton University Press, Princeton, NJ, 1979.

[5] V. Guillemin \& S. Sternberg, On the equations of motion of a classical particle in a Yang-Mills field and the principal of general covariance, Hadronic J. 1 (1978) 1-32.

[6] _ The moment map and collective motion, Ann. Physics. 127 (1980) 220-253.

[7] _ Moments and reductions, Diff. Geom. Meth. in Math. Phys. Proc. (Clausthal, 1980), Lecture Notes in Math. Vol. 905, Springer, Berlin, 1982, 52-65.

[8] The Frobenius reciprocity theorem from the symplectic point of view, Diff. Geom. Methods in Math. Phys. Proc., Clausthal, 1981.

[9] Convexity properties of the moment map, Invent. Math. 67 (1982) 491-513.

[10] Geometric quantization and multiplicities of group representations, Invent. Math. 67 (1982) 515-538.

[11] Homogeneous quantization and multiplicities of group representations, J. Funct. Anal. 47 (1982) 344-380.

[12] On collective complete integrability according to the method of Thimm, Ergodic Theory Dynamical Systems, to appear.

[13] S. Helgason, Differential geometry and symmetric spaces, Academic Press, New York, 1962.

[14] J. Humphreys, Introduction to Lie algebras and representation theory, Springer, New York, 1972.

[15] D. Kazhdan, B. Kostant \& S. Sternberg, Hamiltonian group actions and dynamical systems of Calogero type, Comm. Pure Appl. Math. 31 (1978) 481-507.

[16] F. Kirwan, The cohomology of quotient spaces in algebraic geometry, Thesis, Oxford, 1982.

[17] B. Kostant, Proceedings of the Clalusthal Conference in Mathematical Physics (July 1981), to appear.

[18] M. Kramer, Spharische Untergruppen in kompacten zusammenhangenden Lie Gruppen, Compositio Math. 38 (1979) 129-153. 
[19] J. Marsden \& A. Weinstein, Reduction of symplectic manifolds with symmetry, J. Math. Phys. 5 (1974) 121-130.

[20] A. S. Mishchenko \& A. T. Fomenko, A generalized Liouville method for the integration of Hamiltonian systems, Funktsional Anal. i Priložen 12 (1978) 46-56.

[21] L. Ness, A stratification of the null cone via the moment map, Amer. J. Math., to appear.

[22] E. A. Planchart, Analogies in symplectic geometry of some results of Cartan in representation theory, Thesis, Berkeley, 1982.

[23] S. Sternberg, Minimal coupling and the symplectic mechanics of a classical particle in the presence of a Yang-Mills field, Proc. Nat. Acad. Sci. U.S.A. 74 (1977) 5253-5254.

[24] S. Sternberg \& T. Ungar, Classical and prequantized mechanics without Lagrangians or Hamiltonians, Hadronic J. 1 (1978) 33-45.

[25] A. Weinstein, Lectures on symplectic manifolds, CBMS Regional Conference Series in Math. No. 29, Amer. Math. Soc., Providence, RI, 1977.

[26] _ A universal phase space for particles in Yang-Mills fields, Lett. Math. Phys. 2 (1977/78) 417-420.

[27] __ Fat bundles and symplectic manifolds, Advances in Math. 37 239-250.

[28] _ Symplectic geometry, Bull. Amer. Math. Soc. (N.S.) 5 (1981) 1-13.

[29] N Neighborhood classification of isotropic embedding, J. Differential Geometry 16 (1981) 125-128.

[30]__ The local structure of Poisson manifolds, Berkeley, 1982 (preprint).

HARVARD UNIVERSITY 